\title{
EXACT CONTROLLABILITY FOR A NONLINEAR STOCHASTIC WAVE EQUATION
}

BUI AN TON

Received 2 July 2005; Accepted 7 September 2005

The exact controllability for a semilinear stochastic wave equation with a boundary control is established. The target and initial spaces are $L^{2}(G) \times H^{-1}(G)$ with $G$ being a bounded open subset of $R^{3}$ and the nonlinear terms having at most a linear growth.

Copyright (C 2006 Bui An Ton. This is an open access article distributed under the Creative Commons Attribution License, which permits unrestricted use, distribution, and reproduction in any medium, provided the original work is properly cited.

\section{Introduction}

Let $(\Omega, \mathcal{A}, P)$ be a probability space with $\Omega$ being completely regular and let $w$ be a standard Wiener process on the space. Let $G$ be a bounded open subset of $R^{3}$ with a smooth boundary and consider the initial boundary value problem

$$
\begin{gathered}
d y_{1}=y_{2} d t \quad \text { in } Q \text { a.s., } \\
d y_{2}-\Delta y_{1} d t-d u=f\left(t, y_{1}\right) d w+g\left(t, y_{1}\right) d t \quad \text { in } Q \text { a.s., } \\
y_{1}(\cdot, t, \omega)=0 \quad \text { on } \Gamma_{0} \times(0, T) \text { a.s., } \\
y_{1}(\cdot, t, \omega)=v \quad \text { on } \Gamma_{1} \times(0, T) \text { a.s., } \\
\mathbf{y}(\cdot, 0, \omega)=\boldsymbol{\alpha}=\left\{\alpha_{0}, \alpha_{1}\right\} \quad \text { in } G \text { a.s. }
\end{gathered}
$$

The functions $f, g$ are in $C^{1}(R)$ with $f^{\prime}, g^{\prime}$ in $L^{\infty}(R)$ and

$$
Q=G \times(0, T), \quad \partial G=\Gamma_{0} \bigcup \Gamma_{1}, \quad \Gamma_{0} \bigcap \Gamma_{1}=\varnothing, \quad \Gamma_{1} \neq \varnothing
$$

The given surface $\Gamma_{1}$ is closed. For given $\boldsymbol{\alpha}, \boldsymbol{\beta}$ in $L^{2}(G) \times H^{-1}(G)$, one wishes to find controls:

$$
\{u, v\} \in L^{2}\left(\Omega, \mathscr{A}, P ; C\left(0, T ; L^{2}(G)\right)\right) \times L^{2}\left(\Omega, \mathscr{A}, P ; L^{2}\left(0, T ; L^{2}\left(\Gamma_{1}\right)\right)\right)
$$


such that a solution $y=\left(y_{1}, y_{2}\right)$ of (1.1) takes the value

$$
y(\cdot, T, \omega)=\boldsymbol{\beta} \text { in } G \text { a.s. }
$$

for some large $T>T_{0}$.

The exact controllability of deterministic linear wave equations has been extensively investigated both theoretically and numerically, using the Hilbert uniqueness method of Lions [4].The problem arises in applied sciences when one wishes for example to steer a large complex structure to a specified target (cf. Lions [4], Russell [5]). The result was extended by Zuazua [8] to semilinear wave equations when the initial and target spaces are $H^{\gamma}(G) \times H^{-1+\gamma}(G)$ for some $\gamma>0$. Using the notion of accretive mapping, the author has established in [6], the exact controllability of a semilinear wave equation with at most a linear growth in the nonlinear term, the initial and target spaces being the natural ones, that is, $L^{2}(G) \times H^{-1}(G)$. The literature on the exact controllability of the stochastic wave equation seems scarce. In [7], the author has shown the exact controllability of (1.1)(1.4) when the white noise is independent of the state and it is the purpose of this paper to treat the general case with $f$ depending on $y_{1}$.

\section{Notations, assumption and some preliminary results}

Throughout the paper we will assume that $\Omega$ is completely regular and by abuse of notations, we will denote by $(\cdot, \cdot)$ the $L^{2}(G)$ inner product as well as the pairings of $H_{0}^{1}(G)$ with its dual $H^{-1}(G)$.

Assumption 2.1. Let $f, g$ be $C^{1}((0, T) \times R)$ functions. We assume that

$$
\begin{gathered}
\left\|\frac{\partial f}{\partial \xi}(t, \cdot)\right\|_{L^{\infty}(R)}, \quad\left\|\frac{\partial g}{\partial \xi}(t, \cdot)\right\|_{L^{\infty}(R)} \leq C, \quad \forall t \in[0, T], \\
|f(t, y)|+|g(t, y)| \leq C\{1+|y|\}, \quad \forall\{t, y\} \in(0, T) \times R .
\end{gathered}
$$

With initial and target spaces in $L^{2}(G) \times H^{-1}(G)$, approximating solutions of the linearized version of (1.1)-(1.4) do not have enough regularity to allow the use of a compactness argument for the nonlinear terms $f$ and $g$. The difficulty is circumvented by using an argument of the theory of monotone operators involving accretive mappings introduced by Browder [2], Kato [3].

Let $J$ be the duality mapping of $L^{2}\left(0, T ; H^{-1}(G)\right)$ into its dual, $L^{2}\left(0, T ; H_{0}^{1}(G)\right)$, with gauge function $\Phi(r)=r$. Then it is known that

$$
\begin{aligned}
\int_{0}^{T}(J y, y) d t & =\|y\|_{L^{2}\left(0, T ; H^{-1}(G)\right)}\|J y\|_{L^{2}\left(0, T ; H_{0}^{1}(G)\right)} \\
& =\|y\|_{L^{2}\left(0, T ; H^{-1}(G)\right)}^{2}, \quad \forall y \in L^{2}\left(0, T ; H^{-1}(G)\right) .
\end{aligned}
$$

Moreover $J$ is monotone and is continuous from the strong topology of $L^{2}(0, T$; $\left.H^{-1}(G)\right)$ to the weak topology of $L^{2}\left(0, T ; H_{0}^{1}(G)\right)$. 
Let $F$ be a mapping of $L^{2}\left(0, T ; H^{-1}(G)\right)$ into $L^{2}\left(0, T ; H^{-1}(G)\right)$ with $D(F)=L^{2}(0, T$; $\left.L^{2}(G)\right)$. The mapping $F$ is said to be accretive with respect to $J$ if

$$
\int_{0}^{T}(F(y)-F(z), J(y-z)) d t \geq 0, \quad \forall y, z \in D(F) .
$$

Lemma 2.2. Let $f$ be in $C^{1}((0, T) \times R)$ and suppose that Assumption 2.1 is satisfied. Then $\lambda I+f$ is accretive with respect to the duality mapping $J$ for large, $\lambda>\lambda_{0}$.

Proof. We have

$$
\begin{aligned}
\int_{0}^{T}(f(t, y)-f(t, z), J(y-z)) d t & =\int_{0}^{T}\left((y-z) f^{\prime}(t, \xi), J(y-z)\right) d t \\
& \leq\left\|(y-z) f^{\prime}\right\|_{L^{2}\left(0, T ; H^{-1}(G)\right)}\|J(y-z)\|_{L^{2}\left(0, T ; H_{0}^{1}(G)\right)} \\
& \leq\left\|(y-z) f^{\prime}\right\|_{L^{2}\left(0, T ; H^{-1}(G)\right)}\|y-z\|_{L^{2}\left(0, T ; H^{-1}(G)\right)}
\end{aligned}
$$

for all $y, z$ in $L^{2}\left(0, T ; L^{2}(G)\right)$. On the other hand, it is clear that

$$
\begin{aligned}
\left\|(y-z) f^{\prime}\right\|_{L^{2}\left(0, T ; H^{-1}(G)\right)} & =\inf _{\|\varphi\|_{L^{2}\left(0, T ; H_{0}^{1}(G)\right)}}\left|\int_{0}^{T}\left((y-z) f^{\prime}, \varphi\right) d t\right| \\
& \leq\left\|f^{\prime}\right\|_{L^{\infty}((0, T) \times R)} \int_{0}^{T}(|y-z|,|\varphi|) d t \\
& \leq\left\|f^{\prime}\right\|_{L^{\infty}((0, T) \times R)}\|y-z\|_{L^{2}\left(0, T ; H^{-1}(G)\right)}\|\varphi\|_{L^{2}\left(0, T ; H_{0}^{1}(G)\right)}
\end{aligned}
$$

for all $y, z$ in $L^{2}\left(0, T ; L^{2}(G)\right)$. Thus,

$$
\begin{aligned}
& \left\|(y-z) f^{\prime}\right\|_{L^{2}\left(0, T ; H^{-1}(G)\right)} \\
& \quad \leq\left\|f^{\prime}\right\|_{L^{\infty}((0, T) \times R)}\|y-z\|_{L^{2}\left(0, T ; H^{-1}(G)\right)}, \quad \forall y, z \in L^{2}\left(0, T ; L^{2}(G)\right) .
\end{aligned}
$$

Take $\lambda>\left\|f^{\prime}\right\|_{L^{\infty}((0, T) \times R)}$ and we have

$$
\begin{aligned}
\int_{0}^{T}(( & \lambda I+f)(y-z), J(y-z)) d t \\
& \geq \lambda\|y-z\|_{L^{2}\left(0, T ; H^{-1}(G)\right)}^{2}-\left|\int_{0}^{T}(f(y)-f(z), J(y-z)) d t\right| \\
& \geq\left\{\lambda-\left\|f^{\prime}\right\|_{L^{\infty}((0, T) \times R)}\right\}\|y-z\|_{L^{2}\left(0, T ; H^{-1}(G)\right)}^{2} \\
& \geq 0, \quad \forall y, z \in L^{2}\left(0, T ; L^{2}(G)\right) .
\end{aligned}
$$

The lemma is proved.

The following result has been shown by the author in [6].

Lemma 2.3. Let $f$ be as in Assumption 2.1 and suppose that

$$
y_{n} \longrightarrow y \quad \text { in }\left(L^{2}\left(0, T ; L^{2}(G)\right)\right)_{\text {weak }} \cap L^{2}\left(0, T ; H^{-1}(G)\right) \text {, }
$$


then

$$
f\left(t, y_{n_{j}}\right) \longrightarrow f(t, y) \quad \text { in }\left(L^{2}\left(0, T ; L^{2}(G)\right)\right)_{\text {weak }}
$$

With $f$ as in Assumption 2.1, it follows from Lemma 2.2 that $\lambda I+f$ is accretive with respect to the duality mapping $J$. Since

$$
\begin{gathered}
J\left(y_{n}\right) \longrightarrow J(y) \quad \text { weakly in } L^{2}\left(0, T ; H_{0}^{1}(G)\right), \\
\left\|J\left(y_{n}\right)\right\|_{L^{2}\left(0, T ; H_{0}^{1}(G)\right)}=\left\|y_{n}\right\|_{L^{2}\left(0, T ; H^{-1}(G)\right)} \longrightarrow\|y\|_{L^{2}\left(0, T ; H^{-1}(G)\right)}=\|J(y)\|_{L^{2}\left(0, T ; H_{0}^{1}(G)\right)}
\end{gathered}
$$

and since $L^{2}\left(0, T ; H_{0}^{1}(G)\right)$ is a Hilbert space, we have

$$
J\left(y_{n}\right) \longrightarrow J(y) \quad \text { in } L^{2}\left(0, T ; H_{0}^{1}(G)\right) .
$$

Now using an argument of the theory of monotone operators applied to accretive mappings, the author has shown in [6] the weak convergence in $L^{2}\left(0, T ; L^{2}(G)\right)$ of the sequence $\left\{f\left(t, y_{n_{j}}\right)\right\}$.

\section{Main result}

The main result of the paper is the following theorem.

Theorem 3.1. Let $(\Omega, \mathcal{A}, P)$ be a probability space with $\Omega$ being completely regular and let $w$ be a standard Wiener process on the space. Let $f, g$ be as in Assumption 2.1 and let $\boldsymbol{\alpha}, \boldsymbol{\beta}$ be in $L^{2}(G) \times H^{-1}(G)$. Then for $T>T_{0}$, there exists a weak solution $\{u, v, y\}$ in

$$
\begin{aligned}
L^{2}(\Omega, \mathscr{A}, P & \left.; C\left(0, T ; L^{2}(G)\right)\right) \times L^{4}\left(\Omega, \mathscr{A}, P ; L^{2}\left(0, T ; L^{2}\left(\Gamma_{1}\right)\right)\right) \\
& \times L^{4}\left(\Omega, \mathcal{A}, P ; C\left(0, T ; L^{2}(G)\right)\right) \times L^{4}\left(\Omega, \mathcal{A}, P ; C\left(0, T ; H^{-1}(G)\right)\right),
\end{aligned}
$$

of (1.1)-(1.4). Moreover,

$$
E\left(\left\|y_{1}\right\|_{C\left(0, T ; L^{2}(G)\right)}^{4}\right)+E\left(\left\|y_{2}\right\|_{C\left(0, T ; H^{-1}(G)\right)}^{4}\right)+E\left(\|v\|_{L^{2}\left(0, T ; L^{2}\left(\Gamma_{1}\right)\right)}^{4}\right) \leq C(1+\mathscr{E}(\boldsymbol{\alpha}, \boldsymbol{\beta}))
$$

with

$$
\mathscr{E}(\boldsymbol{\alpha}, \boldsymbol{\beta})=\|\boldsymbol{\alpha}\|_{L^{2}(G) \times H^{-1}(G)}^{4}+\|\boldsymbol{\beta}\|_{L^{2}(G) \times H^{-1}(G)}^{4} .
$$

The proof of the theorem can be split into three steps.

Step 1. Let $\hat{\mathbf{y}}$ be in

$$
L^{4}\left(\Omega, \mathscr{A}, P ; C\left(0, T ; L^{2}(G)\right)\right) \times L^{4}\left(\Omega, \mathscr{A}, P ; C\left(0, T ; H^{-1}(G)\right)\right)
$$

and consider the exact controllability of the linear wave equation

$$
\begin{gathered}
d z_{1}=z_{2} d t \quad \text { in } Q, \\
d z_{2}-\Delta z_{1} d t=0 \quad \text { in } Q, \\
z_{1}=0 \quad \text { on } \Gamma_{0} \times(0, T), \quad z_{1}=v \quad \text { on } \Gamma_{1} \times(0, T), \\
\mathbf{z}(\cdot, 0)=\boldsymbol{\alpha}, \quad \mathbf{z}(\cdot, T)=\boldsymbol{\beta}-\hat{\mathbf{y}}(\cdot, T) \quad \text { in } G .
\end{gathered}
$$


The following result is well known.

Lemma 3.2. Suppose all the hypotheses of Theorem 3.1 are satisfied, let $\hat{y}$ be in $C(0, T$; $\left.L^{2}(G)\right) \times C\left(0, T ; H^{-1}(G)\right)$. Then for $T>T_{0}$, there exists $\{v, \mathbf{z}\}$ in

$$
L^{2}\left(0, T ; L^{2}\left(\Gamma_{1}\right)\right) \times C\left(0, T ; L^{2}(G)\right) \times C\left(0, T ; H^{-1}(G)\right),
$$

weak solution of (3.5). Moreover,

$$
\begin{aligned}
& \|v\|_{L^{2}\left(0, T ; L^{2}\left(\Gamma_{1}\right)\right)}+\left\|z_{1}\right\|_{C\left(0, T ; L^{2}(G)\right)}+\left\|z_{2}\right\|_{C\left(0, T ; H^{-1}(G)\right)} \\
& \quad \leq C\left\{1+\|\boldsymbol{\alpha}\|_{L^{2}(G) \times H^{-1}(G)}+\|\boldsymbol{\beta}\|_{L^{2}(G) \times H^{-1}(G)}+\|\hat{\boldsymbol{y}}(\cdot, T)\|_{L^{2}(G) \times H^{-1}(G)}\right\} .
\end{aligned}
$$

The constant $C$ is independent of $\hat{\mathbf{y}}, \boldsymbol{\alpha}, \boldsymbol{\beta}$.

We will now consider the case when $\hat{y}$ is in

$$
L^{4}\left(\Omega, \mathscr{A}, P ; C\left(0, T ; L^{2}(G)\right)\right) \times L^{4}\left(\Omega, \mathscr{A}, P ; C\left(0, T ; H^{-1}(G)\right)\right)
$$

Let

$$
S_{\hat{y}}=\{\{v, \mathbf{z}\}:\{v, \mathbf{z}\} \text { solution of }(3.5)\}
$$

It follows from Lemma 3.2 that $S_{\hat{y}}$ is nonempty.

Lemma 3.3. Let $S_{\hat{y}}$ be as in (3.9), then it is a closed, bounded convex subset of $L^{2}(0, T$; $\left.L^{2}\left(\Gamma_{1}\right)\right) \times L^{2}\left(0, T ; L^{2}(G)\right) \times L^{2}\left(0, T ; H^{-1}(G)\right)$.

Proof. Since the problem (3.5) is linear, it is clear that $S_{\hat{\mathrm{y}}}$ is a convex subset of $L^{2}(0, T$; $\left.L^{2}\left(\Gamma_{1}\right)\right) \times L^{2}\left(0, T ; L^{2}(G)\right) \times L^{2}\left(0, T ; H^{-1}(G)\right)$. It follows from Lemma 3.2 that the set is bounded. Suppose that

$$
\left\{v_{n}, \mathbf{z}_{n}\right\} \longrightarrow\{v, \mathbf{z}\}
$$

in

$$
L^{2}\left(0, T ; L^{2}\left(\Gamma_{1}\right)\right) \times L^{2}\left(0, T ; L^{2}(G)\right) \times L^{2}\left(0, T ; H^{-1}(G)\right)
$$

with $\left\{v_{n}, \mathbf{z}_{n}\right\} \in S_{\hat{\mathbf{y}}}$. It is trivial to check that indeed $\{v, \mathbf{z}\} \in S_{\hat{\mathbf{y}}}$.

Let $\pi_{S_{\hat{y}}}$ be the projection of

$$
L^{2}\left(0, T ; L^{2}\left(\Gamma_{1}\right)\right) \times L^{2}\left(0, T ; L^{2}(G)\right) \times L^{2}\left(0, T ; H^{-1}(G)\right)
$$

onto the closed convex set $S_{\hat{y}}$, defined by

$$
\left\|||\{, \boldsymbol{x}\}-\pi_{S_{\hat{y}}}(\{v, \boldsymbol{x}\})\right\| \|=\inf \left\{\|||\{v, \boldsymbol{x}\}-\{\hat{v}, \hat{\boldsymbol{x}}\}\|: \forall\{\hat{v}, \hat{\boldsymbol{x}}\} \in S_{\hat{\mathbf{y}}}\right\}
$$

with

$$
\|\|\{v, \boldsymbol{x}\}\|\|=\|\{v, \boldsymbol{x}\}\|_{L^{2}\left(0, T ; L^{2}\left(\Gamma_{1}\right)\right) \times L^{2}\left(0, T ; L^{2}(G)\right) \times L^{2}\left(0, T ; H^{-1}(G)\right)} .
$$


Then $\pi_{S_{\widehat{y}}}$ is uniquely defined and let

$$
\{\tilde{v}, \bar{z}\}=\pi_{S_{\hat{y}}}(0)
$$

be the unique element of minimal $L^{2}\left(0, T ; L^{2}\left(\Gamma_{1}\right)\right) \times L^{2}\left(0, T ; L^{2}(G)\right) \times L^{2}\left(0, T ; H^{-1}(G)\right)$ norm of $S$.

Let

$$
\Lambda(\hat{\boldsymbol{y}})=\{\{\tilde{v}, \bar{z}\}:\{\tilde{v}, \bar{z}\} \text { solution of }(3.5) \text { as in }(3.15)\}
$$

then it maps

$$
\begin{aligned}
C\left(0, T ; L^{2}(G)\right) \times C\left(0, T ; H^{-1}(G)\right) & \\
& \longrightarrow L^{2}\left(0, T ; L^{2}\left(\Gamma_{1}\right)\right) \times C\left(0, T ; L^{2}(G)\right) \times C\left(0, T ; H^{-1}(G)\right) .
\end{aligned}
$$

Lemma 3.4. Let $\Lambda$ be as in (3.16), then its graph is closed.

Proof. It is an immediate consequence of Lemma 3.2.

Let $\Phi(\omega)$ be the mapping

$$
\Phi(\omega)=\{\hat{\mathbf{y}}(\cdot, \omega)\}
$$

of $\Omega$ into $C\left(0, T ; L^{2}(G)\right) \times C\left(0, T ; H^{-1}(G)\right)$.

Set

$$
\Theta(\omega)=\Lambda \circ \Phi(\omega)=\{\{\tilde{v}(\cdot, \omega), \bar{z}(\cdot, \omega)\} \text { : solution of }(3.5) \text { as in }(3.15)\} .
$$

Lemma 3.5. The mapping $\Lambda$ has a universally measurable section $\sigma$ and the application $\sigma \circ \Theta$ of

$$
\Omega \longrightarrow L^{2}\left(0, T ; L^{2}\left(\Gamma_{1}\right)\right) \times C\left(0, T ; L^{2}(G)\right) \times C\left(0, T ; H^{-1}(G)\right)
$$

is a measurable section of $\Theta$. Furthermore

$$
\begin{aligned}
& E\left(\left\|z_{1}\right\|_{C\left(0, T ; L^{2}(G)\right)}^{2}\right)+E\left(\left\|z_{2}\right\|_{C\left(0, T ; H^{-1}(G)\right)}^{2}\right)+E\left(\|\tilde{v}\|_{L^{2}\left(0, T ; L^{2}\left(\Gamma_{1}\right)\right)}^{2}\right) \\
& \quad \leq C\left\{1+\|\boldsymbol{\alpha}\|_{L^{2}(G) \times H^{-1}(G)}^{2}+\|\boldsymbol{\beta}\|_{L^{2}(G) \times H^{-1}(G)}^{2}+E\left(\|\hat{\boldsymbol{y}}(\cdot, T)\|_{L^{2}(G) \times H^{-1}(G)}^{2}\right)\right\} .
\end{aligned}
$$

Proof. The mapping $\Lambda$ has nonempty closed images in $L^{2}\left(0, T ; L^{2}\left(\Gamma_{1}\right)\right) \times C\left(0, T ; L^{2}(G)\right) \times$ $C\left(0, T ; H^{-1}(G)\right)$ with a closed graph. It follows from a theorem of Von Neumann that there exists a universally measurable section $\sigma$ of $\Lambda$ (cf. [1, Theorem 3.1]).

Since $P$ is a Radon measure on a completely regular space and since $\Phi$ is a random variable and therefore measurable in the sense of Lusin, we deduce that for each positive integer $k$ there exists a compact set $K_{k}$ of $\Omega$ such that

$$
P\left(\frac{\Omega}{K_{k}}\right) \leq \frac{1}{k}
$$

and the restriction $\Phi_{k}=\left.\Phi\right|_{K_{k}}$ of $\Phi$ to $K_{k}$ is continuous. 
We may assume that $\left\{K_{k}\right\}$ is an increasing sequence.

The measure $P$ induces on $K_{k}$ a Radon measure $P_{k}$ and $\Phi_{k}\left(P_{k}\right)$ is a Radon measure on $L^{2}\left(0, T ; L^{2}\left(\Gamma_{1}\right)\right) \times C\left(0, T ; L^{2}(G)\right) \times C\left(0, T ; H^{-1}(G)\right)$. Since $\sigma$ is $\Phi_{k}\left(P_{k}\right)$ measurable, it follows that $\sigma \circ \Phi_{k}$ is $P$ measurable on $K_{k}$ in $L^{2}\left(0, T ; L^{2}\left(\Gamma_{1}\right)\right) \times C\left(0, T ; L^{2}(G)\right)$ $\times C\left(0, T ; H^{-1}(G)\right)$. Let

$$
\left\{\widetilde{v}_{k}(\cdot, \omega), \overline{\mathbf{z}}_{k}(\cdot, \omega)\right\}= \begin{cases}(\sigma \circ \Phi)(\cdot, \omega), & \text { if } \omega \in K_{k}, \\ 0, & \text { if } \omega \notin K_{k}\end{cases}
$$

The functions $\left\{\widetilde{v}_{k}(\cdot, \omega), \overline{\mathbf{z}}_{k}(\cdot \omega)\right\}$ from $\Omega$ into $L^{2}\left(0, T ; L^{2}\left(\Gamma_{1}\right)\right) \times C\left(0, T ; L^{2}(G)\right) \times C(0, T$; $\left.H^{-1}(G)\right)$ are measurable. Since $P\left(\bigcup_{k} K_{k}\right)=1$, we have

$$
\left\{\tilde{v}_{k}, \overline{\mathbf{z}}_{k}\right\} \longrightarrow\{\tilde{v}, \overline{\mathbf{z}}\}, \quad \text { a.s. with }\{\tilde{v}, \overline{\mathbf{z}}\} \in \Lambda(\hat{\mathbf{y}})
$$

Thus, $\{\tilde{v}, \overline{\mathbf{z}}\}$ is measurable in the sense of Lusin and therefore is a random variable. The stated estimate is an immediate consequence of that of Lemma 3.2.

Step 2. We now consider the linear stochastic system

$$
\begin{gathered}
d y_{1}=y_{2} d t \quad \text { in } Q \text { a.s., } \\
d y_{2}-\Delta y_{1} d t=\left\{f\left(t, \bar{z}_{1}+\hat{y}_{1}\right)-f\left(t, \bar{z}_{1}\right)\right\} d w \\
+\left\{g\left(t, \bar{z}_{1}+\hat{y}_{1}\right)-g\left(t, \bar{z}_{1}\right)\right\} d t \quad \text { in } Q \text { a.s., } \\
y_{1}=0 \quad \text { on } \partial G \times(0, T) \text { a.s., } \\
\mathbf{y}(\cdot, 0)=0 \quad \text { in } G \text { a.s. }
\end{gathered}
$$

Lemma 3.6. Suppose all the hypotheses of Theorem 3.1 are satisfied and let $\overline{\mathbf{z}}$ be as in (3.15). Then there exists a unique y, solution of (3.25). Moreover,

$$
\begin{aligned}
& E\left(\|\mathbf{y}(\cdot, t)\|_{L^{2}(G)}^{4}\right)+E\left(\left\|y_{1}\right\|_{L^{2}\left(0, t ; H_{0}^{1}(G)\right)}^{4}\right) \\
& \quad \leq C\left\{1+\exp \left\{E\left(\left\|\hat{y}_{1}\right\|_{L^{2}\left(0, t ; L^{2}(G)\right)}^{4}\right)\right\}\right\}, \quad \forall t \in[0, T] .
\end{aligned}
$$

Furthermore,

$$
\begin{gathered}
E\left(\left\|y_{1}^{\prime}\right\|_{L^{2}\left(0, T ; L^{2}(G)\right)}^{2}\right) \leq C\left\{1+\exp \left\{E\left(\left\|\hat{y}_{1}\right\|_{L^{2}\left(0, T ; L^{2}(G)\right.}^{2}\right)\right\}\right\}, \\
E\left(\sup _{n} \frac{1}{v_{n}} \sup _{|s| \leq \delta_{n}} \int_{0}^{t}\left\|y_{2}(r+s)-y_{2}(r)\right\|_{H^{-1}(G)}^{2} d r\right) \\
\leq C\left\{1+\mathscr{E}(\boldsymbol{\alpha}, \boldsymbol{\beta})+\exp \left(E\left(\left\|\hat{y}_{1}\right\|_{L^{2}\left(0, t ; L^{2}(G)\right)}^{4}\right)\right)\right\}
\end{gathered}
$$

with $\left\{v_{n}, \delta_{n}\right\} \rightarrow 0^{+}$and $\sum_{n} \sqrt{\delta_{n}} / \nu_{n}<\infty$. The constant $C$ is independent of $t$ and of $\bar{z}_{1}$.

Proof. The existence of a unique solution of (3.25) is well known. we will now establish the estimate of the lemma. 
(1) A standard argument gives

$$
\begin{aligned}
\|\mathbf{y}(\cdot, t)\|_{L^{2}(G)}^{2}+\left\|y_{1}\right\|_{L^{2}\left(0, t ; H_{0}^{1}(G)\right)}^{2} \leq & \left|\int_{0}^{t}\left(f\left(s, \bar{z}_{1}+\hat{y}_{1}\right)-f\left(\cdot, \bar{z}_{1}\right), y_{2}\right) d w\right| \\
& +\int_{0}^{t}\left\|g\left(s, \bar{z}_{1}+\hat{y}_{1}\right)-g\left(\cdot, \bar{z}_{1}\right)\right\|_{L^{2}(G)}^{2} d s .
\end{aligned}
$$

With $f, g$ as in Assumption 2.1, they are Lipschitz continuous in $L^{2}\left(0, T ; L^{2}(G)\right)$ and thus

$$
\begin{aligned}
& \|\mathbf{y}(\cdot, t)\|_{L^{2}(G)}^{2}+\left\|y_{1}\right\|_{L^{2}\left(0, t ; H_{0}^{1}(G)\right)}^{2} \\
& \quad \leq C \int_{0}^{t}\left\|\hat{y}_{1}\right\|_{L^{2}(G)}^{2} d s+\left|\int_{0}^{t}\left(f\left(\cdot, \bar{z}_{1}+\hat{y}_{1}\right)-f\left(\cdot, \bar{z}_{1}\right), y_{2}\right) d w\right| .
\end{aligned}
$$

Taking the square of the two sides of (3.29) and then the mathematical expectation, we obtain by applying the martingale inequality,

$$
E\left(\|\mathbf{y}(\cdot, t)\|_{L^{2}(G)}^{4}\right) \leq C\left\{E\left(\left\|\hat{y}_{1}\right\|_{L^{2}\left(0, t ; L^{2}(G)\right)}^{4}\right)+\int_{0}^{t} E\left(\left\|y_{2}\right\|_{L^{2}(G)}^{4}\right) d s\right\} .
$$

We have used the hypothesis that $f$ is $L^{2}\left(0, T ; L^{2}(G)\right)$ Lipschitz continuous. An application of the Gronwall lemma yields

$$
E\left(\|\mathbf{y}(\cdot, t)\|_{L^{2}(G)}^{4}\right) \leq C\left\{1+\exp \left(E\left(\left\|\hat{y}_{1}\right\|_{L^{2}\left(0, t ; L^{2}(G)\right)}^{4}\right)\right)\right\}
$$

for all $t \in[0, T]$. The constant $C$ is independent of $\bar{z}_{1}$. Now going back to (3.30) and we obtain

$$
E\left(\left\|y_{1}\right\|_{L^{2}\left(0, t ; H_{0}^{1}(G)\right)}^{4}\right) \leq C\left\{1+\exp \left\{E\left(\left\|\hat{y}_{1}\right\|_{L^{2}\left(0, t ; L^{2}(G)\right)}^{4}\right)\right\}\right\}
$$

for all $t \in[0, T]$. The constant $C$ is independent of $\bar{z}_{1}$. It is clear that

$$
E\left(\left\|y_{1}^{\prime}\right\|_{L^{2}\left(0, T ; L^{2}(G)\right)}^{2}\right) \leq C\left\{1+\exp \left\{E\left(\left\|\hat{y}_{1}\right\|_{L^{2}\left(0, T ; L^{2}(G)\right)}^{2}\right)\right\}\right\} .
$$

(2) For any positive, $\theta$ we have

$$
\begin{aligned}
\left\|y_{2}(t+\theta)-y_{2}(t)\right\|_{H^{-1}(G)} & \\
\leq & C \theta+\int_{t}^{t+\theta}\left\|y_{1}\right\|_{H_{0}^{1}(G)} d s+\left\|\int_{t}^{t+\theta}\left\{f\left(s, \bar{z}_{1}+\hat{y}_{1}\right)-f\left(s, \bar{z}_{1}\right)\right\} d w\right\|_{L^{2}(G)} \\
& +C \int_{t}^{t+\theta}\left\|\hat{y}_{1}\right\|_{L^{2}(G)} d s .
\end{aligned}
$$

Taking the square of the two sides, integrating with respect to $t$ from 0 to $T$ and then the mathematical expectation, we obtain by applying the martingale inequality,

$$
\begin{aligned}
& E\left(\sup _{|\theta| \leq \delta} \int_{0}^{T}\left\|y_{2}(t+\theta)-y_{2}(t)\right\|_{H^{-1}(G)}^{2} d t\right) \\
& \quad \leq C \delta^{2}+C \delta^{2} E\left(\int_{0}^{T}\left\|y_{1}\right\|_{H_{0}^{1}(G)}^{2} d t\right)+C \delta E\left(\int_{0}^{T}\left\|\hat{y}_{1}\right\|_{L^{2}(G)}^{2} d t\right) \leq C_{1} \delta .
\end{aligned}
$$


We have used the hypothesis that $f$ and $g$ are $L^{2}\left(0, T ; L^{2}(G)\right)$ Lipschitz continuous. Taking into account the estimates of the first part, we get the stated result.

Step 3. Let

$$
\begin{aligned}
\mathscr{B}= & \left\{\left(y_{1}, y_{2}\right): E\left(\|\mathbf{y}(\cdot, t)\|_{L^{2}(G)}^{4}\right), E\left(\left\|y_{1}^{\prime}\right\|_{L^{2}\left(0, t ; L^{2}(G)\right)}^{2}\right) \leq C \exp (t),\right. \\
& \left.E\left(\sup _{|\theta| \leq \delta} \int_{0}^{t}\left\|y_{2}(r+\theta)-y_{2}(r)\right\|_{H^{-1}(G)}^{2} d r\right) \leq C \delta, \forall t \in[0, T]\right\} .
\end{aligned}
$$

Let $\mathbf{h}$ be an element of

$$
L^{2}\left(\Omega, \mathscr{A}, P ; L^{2}\left(0, T ; H_{0}^{1}(G)\right)\right) \times L^{2}\left(\Omega, \mathscr{A}, P ; L^{2}(G)\right) .
$$

Since $\Omega$ is completely regular and $\mathbf{h}$ is a random variable and hence is measurable in the Lusin, for each $k$ there exists a compact subset $K_{k}$ of $\Omega$ such that

$$
P\left(\frac{\Omega}{K_{k}}\right) \leq \frac{1}{k}
$$

and the restriction of $\mathbf{h}$ to $K_{k}$ is continuous on $K_{k}$ with values in $H_{0}^{1}(G) \times L^{2}(G)$, we may assume without loss of generality that the $K_{k}$ 's are increasing.

LEMMA 3.7. Let $\mathscr{B}$ be as above, then it is a compact convex subset of

$$
L^{2}\left(K_{k}, \mathcal{A}, P ; C\left(0, T ; L^{2}(G)\right)\right) \times L^{2}\left(K_{k}, \mathcal{A}, P ; C\left(0, T ; H^{-1}(G)\right)\right) .
$$

Proof. (1) Let $\mathbf{y}_{n}$ be in $\mathscr{B}$, then

$$
\begin{gathered}
E\left(\left\|\mathbf{y}_{n}\right\|_{C\left(0, T ; L^{2}(G)\right)}^{4}\right)+E\left(\left\|y_{1, n}\right\|_{L^{2}\left(0, t ; H_{0}^{1}(G)\right)}^{4}\right)+E\left(\left\|y_{1, n}^{\prime}\right\|_{L^{2}\left(0, T ; L^{2}(G)\right)}^{4}\right) \leq C, \\
E\left(\sup _{|\theta| \leq \delta} \int_{0}^{T}\left\|y_{2, n}(t+\theta)-y_{2, n}(t)\right\|_{H^{-1}(G)}^{2} d t\right) \leq C \delta .
\end{gathered}
$$

Since $\mathbf{y}_{n}$ are random variables, they are measurable in the sense of Lusin. By hypothesis, $\Omega$ is completely regular, thus for each positive integer $k$, there exist compact subsets $K_{1, k}^{n}, K_{2, k}^{n}$ of $\Omega$ with

$$
P\left(\frac{\Omega}{K_{1, k}^{n}}\right), P\left(\frac{\Omega}{K_{2, k}^{n}}\right) \leq \frac{1}{k}
$$

and the restrictions of $\mathbf{y}_{n}$ to $K_{1, k}^{n} \times K_{2, k}^{n}$ are continuous with values in $C\left(0, T ; L^{2}(G)\right) \cap$ $L^{2}\left(0, T ; H_{0}^{1}(G)\right) \times C\left(0, T ; L^{2}(G)\right)$. Moreover the sets $K_{1, k}^{n}, K_{2, k}^{n}$ are increasing. Without loss of generality we will assume that

$$
K_{k} \subset K_{j, k}^{n}, \quad j=1,2 .
$$


(2) Since $\mathbf{y}_{n}$ are in $\mathscr{B}$, a simple proof by contradiction gives

$$
\begin{gathered}
\left\|\mathbf{y}_{n}(\cdot, \omega)\right\|_{C\left(0, T ; L^{2}(G)\right)}+\left\|y_{1, n}(\cdot, \omega)\right\|_{L^{2}\left(0, T ; H_{0}^{1}(G)\right)} \\
+\left\|y_{1, n}^{\prime}(\cdot, \omega)\right\|_{L^{2}\left(0, T ; L^{2}(G)\right)} \leq C_{k}(\omega), \quad \text { a.s. in } K_{k}, \\
\sup _{|\theta| \leq \delta} \int_{0}^{T}\left\|y_{2, n}(t+\theta, \omega)-y_{2, n}(t, \omega)\right\|_{H^{-1}(G)}^{2} d t \leq \delta C_{k}(\omega), \quad \text { a.s. in } K_{k} .
\end{gathered}
$$

Since $K_{k}$ is a compact subset of $\Omega$, we have

$$
K_{k} \subset \bigcup_{j=1}^{N} V_{j}\left(\omega_{j}, \delta\right) \subset \Omega .
$$

It follows from Aubin's theorem that there exists a subsequence such that

$$
y_{1, n_{s}}\left(\cdot, \omega_{j}\right) \longrightarrow y_{1}\left(\cdot, \omega_{j}\right) \quad \text { in } C\left(0, T ; L^{2}(G)\right) .
$$

Furthermore, $y_{1, n} \rightarrow y_{1}$ in

$$
\left(L^{4}\left(\Omega, \mathscr{A}, P ; L^{2}\left(0, T ; H_{0}^{1}(G)\right)\right)\right)_{\text {weak }} \cap\left(L^{4}\left(\Omega, \mathscr{A}, P ; L^{\infty}\left(0, T ; L^{2}(G)\right)\right)\right)_{\text {weak }} \text {. }
$$

From the diagonalization process, we get

$$
y_{1, n}\left(\cdot, \omega_{j}\right) \longrightarrow y_{1}\left(\cdot, \omega_{j}\right) \quad \text { in } C\left(0, T ; L^{2}(G)\right), \forall j .
$$

Since the restriction of $y_{1}$ to $K_{k}$ is continuous with values in $C\left(0, T ; L^{2}(G)\right)$, we have

$$
\begin{aligned}
\int_{K_{k}}\left\|y_{1, n}(\cdot, \omega)-y_{1}(\cdot, \omega)\right\|_{C\left(0, T ; L^{2}(G)\right)}^{2} d P \\
\leq \sum_{j=1}^{N}\left\{\int _ { V _ { j } } \left(\left\|y_{1, n}(\cdot, \omega)-y_{1, n}\left(\cdot, \omega_{j}\right)\right\|_{C\left(0, T ; L^{2}(G)\right)}^{2}\right.\right. \\
\left.\quad+\left\|y_{1, n}\left(\cdot, \omega_{j}\right)-y_{1}\left(\cdot, \omega_{j}\right)\right\|_{C\left(0, T ; L^{2}(G)\right)}^{2}\right) d P \\
\left.+\left\|y_{1}\left(\cdot, \omega_{j}\right)-y_{1}(\cdot, \omega)\right\|_{C\left(0, T ; L^{2}(G)\right)}^{2} d P\right\} \leq C \varepsilon .
\end{aligned}
$$

Hence,

$$
y_{1, n} \longrightarrow y_{1} \quad \text { in } L^{2}\left(K_{k}, \mathscr{A}, P ; C\left(0, T ; L^{2}(G)\right)\right) .
$$

(3) With $y_{2, n}$, we will apply a compactness theorem involving fractional time derivative instead of Aubin's theorem. We get as before as a subsequence such that

$$
\begin{gathered}
y_{2, n}\left(\cdot, \omega_{j}\right) \longrightarrow y_{2}\left(\cdot, \omega_{j}\right) \quad \text { in } C\left(0, T ; H^{-1}(G)\right), j=1, \ldots, N, \\
y_{2, n} \longrightarrow y_{2} \quad \text { in }\left(L^{4}\left(\Omega, \mathscr{A}, P ; L^{\infty}\left(0, T ; L^{2}(G)\right)\right)\right)_{\text {weak* }} .
\end{gathered}
$$

Again by the diagonalization process, we get a subsequence such that

$$
y_{2, n}\left(\cdot, \omega_{j}\right) \longrightarrow y_{2}\left(\cdot, \omega_{j}\right) \quad \text { in } C\left(0, T ; H^{-1}(G)\right), j=1, \ldots, N .
$$


Since $y_{2, n}$ and $y_{2}$ are continuous on $K_{k}$ with values in $C\left(0, T ; H^{-1}(G)\right)$, we have

$$
\begin{aligned}
& \int_{K_{k}}\left\|y_{2, n}(\cdot, \omega)-y_{2}(\cdot, \omega)\right\|_{C\left(0, T ; H^{-1}(G)\right)}^{2} d P \\
& \leq \sum_{j=1}^{N} \int_{V_{j}}\{\left\|y_{2, n}(\cdot, \omega)-y_{2, n}\left(\cdot, \omega_{j}\right)\right\|_{C\left(0, T ; H^{-1}(G)\right)}^{2} \\
&+\left\|y_{2, n}\left(\cdot, \omega_{j}\right)-y_{2}\left(\cdot, \omega_{j}\right)\right\|_{C\left(0, T ; H^{-1}(G)\right)}^{2} \\
&\left.+\left\|y_{2}\left(\cdot, \omega_{j}\right)-y_{2}(\cdot, \omega)\right\|_{C\left(0, T ; H^{-1}(G)\right)}^{2}\right\} d P \leq C \varepsilon .
\end{aligned}
$$

Thus,

$$
\mathbf{y}_{n} \longrightarrow \mathbf{y}_{1} \quad \text { in } L^{2}\left(K_{k}, \mathscr{A}, P ; C\left(0, T ; L^{2}(G)\right)\right) \times L^{2}\left(K_{k}, \mathscr{A}, P ; C\left(0, T ; H^{-1}(G)\right)\right)
$$

and the lemma is proved.

Let $\mathscr{L}$ be the nonlinear mapping of $\mathscr{B}$ into

$$
L^{2}\left(\Omega, \mathcal{A}, P ; C\left(0, T ; L^{2}(G)\right)\right) \times L^{2}\left(\Omega, \mathcal{A}, P ; C\left(0, T ; H^{-1}(G)\right)\right)
$$

defined by

$$
\mathscr{L}(\hat{\mathbf{y}})=\mathbf{y}
$$

where $\mathrm{y}$ is the unique solution of (3.25) given by Lemma 3.6.

Lemma 3.8. The mapping $\mathscr{L}$ takes $\mathscr{B}$ into $\mathscr{B}$.

Proof. It is an immediate consequence of the estimates of Lemma 3.6 and of the Gronwall lemma.

We will consider $\mathscr{B}$ as a subset of

$$
L^{2}\left(K_{k}, \mathcal{A}, P ; C\left(0, T ; L^{2}(G)\right)\right) \times L^{2}\left(K_{k}, \mathcal{A}, P ; C\left(0, T ; H^{-1}(G)\right)\right) .
$$

Lemma 3.9. Let $\mathscr{L}$ be as in (3.70), then $\mathscr{L}$ is continuous from

$$
L^{2}\left(K_{k}, \mathscr{A}, P ; C\left(0, T ; L^{2}(G)\right)\right) \times L^{2}\left(K_{k}, \mathscr{A}, P ; C\left(0, T ; H^{-1}(G)\right)\right)
$$

into itself.

Proof. (1) Let $\hat{\mathbf{y}}_{n} \in \mathscr{B}$ and let $\mathscr{L}\left(\hat{\mathbf{y}}_{n}\right)=\mathbf{y}_{n}$ with $\mathbf{y}_{n}$ being a solution of (3.25). Let $\overline{\mathbf{z}}_{n}$ be the solution of (3.5) with $\hat{\mathbf{y}}$ replaced by $\hat{\mathbf{y}}_{n}$.

Suppose that

$$
\left\{\hat{\mathbf{y}}_{n}, \mathbf{y}_{n}\right\} \longrightarrow\{\hat{\mathbf{y}}, \mathbf{y}\}
$$

in

$$
\left\{L^{2}\left(K_{k}, \mathscr{A}, P ; C\left(0, T ; L^{2}(G)\right)\right) \times L^{2}\left(K_{k}, \mathscr{A}, P ; C\left(0, T ; H^{-1}(G)\right)\right)\right\}^{2} .
$$


We now show that $\mathscr{L}(\hat{\mathbf{y}})=\mathbf{y}$. With $\hat{\mathbf{y}}_{n} \in \mathscr{B}$, it follows from the estimates of Lemmas $3.2,3.5$ that

$$
\begin{aligned}
& E\left(\left\|\bar{z}_{1, n}\right\|_{C\left(0, T ; L^{2}(G)\right)}^{2}\right)+E\left(\left\|\bar{z}_{2, n}\right\|_{C\left(0, T ; H^{-1}(G)\right)}^{2}\right) \\
& \quad+E\left(\left\|\tilde{v}_{n}\right\|_{L^{2}\left(0, T ; L^{2}\left(\Gamma_{1}\right)\right)}^{2}\right)+E\left(\left\|\bar{z}_{1, n}^{\prime}\right\|_{L^{2}\left(0, T ; H^{-1}(G)\right)}\right) \leq C .
\end{aligned}
$$

(2) A proof as done in Lemma 3.7 gives

$$
\bar{z}_{1, n} \longrightarrow \bar{z}_{1} \quad \text { in } L^{2}\left(0, T ; H^{-1}(G)\right) \text {, a.s. in } K_{k},
$$

and $\left\{\bar{z}_{1, n}, \bar{z}_{2, n}, \tilde{v}_{n}\right\} \rightarrow\left\{z_{1}, z_{2}, \tilde{v}\right\}$ in

$$
\begin{aligned}
\left(L^{2}\left(\Omega, \mathscr{A}, P ; L^{\infty}\left(0, T ; L^{2}(G)\right)\right)\right)_{\text {weak }} & \times\left(L^{2}\left(\Omega, \mathscr{A}, P ; L^{\infty}\left(0, T ; H^{-1}(G)\right)\right)\right)_{\text {weak* }} \\
& \times\left(L^{2}\left(\Omega, \mathcal{A}, P ; L^{2}\left(0, T ; L^{2}\left(\Gamma_{1}\right)\right)\right)\right)_{\text {weak }} .
\end{aligned}
$$

(3) An application of Lemma 2.3 gives

$$
\left\{f\left(\cdot, \bar{z}_{1, n}+\hat{y}_{1, n}\right), g\left(\cdot, \bar{z}_{1, n}+\hat{y}_{1, n}\right)\right\} \longrightarrow\left\{f\left(\cdot, \bar{z}_{1}+\hat{y}_{1}\right), g\left(\cdot, \bar{z}_{1}+\hat{y}_{1}\right)\right\}
$$

weakly in $\left(L^{2}\left(0, T ; L^{2}(G)\right)\right)^{2}$, a.s. in $K_{k}$.

(4) We have to show that $\{\bar{v}, \bar{z}\}=\pi_{S_{\hat{y}}}(0)$. It is easy to check that

$$
\bigcap_{n} S_{\widehat{\mathbf{y}}_{n}}=S_{\hat{\mathbf{y}}}
$$

Thus, $S_{\widehat{y}}$ is a closed convex subset of

$$
L^{2}\left(0, T ; L^{2}\left(\Gamma_{1}\right)\right) \times L^{2}\left(0, T ; L^{2}(G)\right) \times L^{2}\left(0, T ; H^{-1}(G)\right) .
$$

We have

$$
\begin{aligned}
\|\{\bar{v}, \bar{z}\}\| \mid & \leq \liminf \left\|\left\{v_{n}, \mathbf{z}_{n}\right\}\right\| \| \\
& \leq\left\|\left\{v_{n}, \mathbf{z}_{n}\right\}\right\| \|, \quad \forall\left\{v_{n}, \mathbf{z}_{n}\right\} \in S_{\widehat{y_{n}}}, n \geq n_{0} \\
& \leq\|\|\{v, \mathbf{z}\} \|, \quad \forall\{v, \mathbf{z}\} \in S_{\widehat{y}} .
\end{aligned}
$$

Therefore $\{\bar{v}, \bar{z}\}=\pi_{S_{\hat{y}}}(0)$.

(5) Since $\mathbf{y}_{n}$ is in $\mathscr{B}$, it is clear that $\mathbf{y} \in \mathscr{B}$. It is now trivial to check that

$$
\mathscr{L}(\hat{\mathbf{y}})=\mathbf{y}
$$

and the lemma is proved.

Lemma 3.10. The mapping $\mathscr{L}$ has a fixed point in $\mathscr{B}$.

Proof. The mapping $\mathscr{L}$ is defined on all of the space

$$
X=L^{2}\left(K_{k}, \mathscr{A}, P ; C\left(0, T ; L^{2}(G)\right)\right) \times L^{2}\left(K_{k}, \mathscr{A}, P ; C\left(0, T ; H^{-1}(G)\right)\right)
$$

and takes $\mathscr{B}$, considered as a subset of $X$, into $\mathscr{B}$. Since $\mathscr{L}$ is continuous from $X$ into $X$, it 
follows from the Schauder fixed point theorem that there exists $\tilde{y} \in \mathscr{B}$ such that

$$
\mathscr{L}(\tilde{\mathbf{y}}(\cdot, \omega))=\tilde{\mathbf{y}}(\cdot, \omega) \quad \text { in } K_{k} .
$$

Proof of Theorem 3.1. From Lemma 3.9, we have

$$
\begin{gathered}
d \bar{z}_{1}=\bar{z}_{2} d t \quad \text { in } Q \text { a.s. in } K_{k}, \\
d \bar{z}_{2}-\Delta \bar{z}_{1} d t=0 \quad \text { in } Q \text { a.s. in } K_{k}, \\
\bar{z}_{1}=0 \quad \text { on } \Gamma_{0} \times(0, T) \text { a.s. in } K_{k}, \\
\bar{z}_{1}=\tilde{v} \quad \text { on } \Gamma_{1} \times(0, T) \text { a.s. in } K_{k}, \\
\overline{\mathbf{z}}(\cdot, 0)=\boldsymbol{\alpha}, \quad \overline{\mathbf{z}}(\cdot, T)=\boldsymbol{\beta}-\tilde{\mathbf{y}}(\cdot, T) \quad \text { in } G \text { a.s. in } K_{k}
\end{gathered}
$$

with

$$
\begin{gathered}
d \tilde{y}_{1}=\tilde{y}_{2} d t \quad \text { in } Q \text { a.s. in } K_{k}, \\
d \tilde{y}_{2}-\Delta \tilde{y}_{1} d t=\left\{f\left(t, \bar{z}_{1}+\tilde{y}_{1}\right)-f\left(t, \bar{z}_{1}\right)\right\} d w \\
+\left\{g\left(t, \bar{z}_{1}+\tilde{y}_{1}\right)-g\left(t, \bar{z}_{1}\right)\right\} d t \quad \text { in } Q \text { a.s. in } K_{k}, \\
\tilde{y}_{1}=0 \quad \text { on } \partial G \times(0, T) \text { a.s. in } K_{k}, \\
\tilde{\mathbf{y}}_{1}(\cdot, 0)=0 \quad \text { in } G \text { a.s. in } K_{k},
\end{gathered}
$$

and $\{\bar{v}, \overline{\mathbf{z}}\}=\pi_{S_{\tilde{y}}}(0)$. Let

$$
\tilde{\mathbf{y}}_{k}(\cdot, \omega)= \begin{cases}\tilde{\mathbf{y}}(\cdot, \omega), & \text { if } \omega \in K_{k}, \\ 0, & \text { if } \omega \notin K_{k}\end{cases}
$$

The same applies for $\overline{\mathbf{z}}_{k}$. Since $P\left(\bigcup_{k} K_{k}\right)=1$, we get

$$
\tilde{\mathbf{y}}_{k} \longrightarrow \tilde{\mathbf{y}}, \quad \text { a.s. in } \Omega
$$

with values in $C\left(0, T ; L^{2}(G)\right) \times C\left(0, T ; H^{-1}(G)\right)$. The same applies for $\overline{\mathbf{z}}_{k}$. Let

$$
\tilde{f}_{k}(\cdot, \omega)= \begin{cases}f\left(\cdot, \tilde{y}_{1}(\cdot, \omega)+\bar{z}_{1}(\cdot, \omega)\right), & \text { if } \omega \in K_{k}, \\ 0, & \text { otherwise }\end{cases}
$$

The $\tilde{f}_{k}$ are measurable functions from $\Omega$ to $L^{2}\left(0, T ; L^{2}(G)\right)$ and since $P\left(\cup K_{k}\right)=1$, we deduce that

$$
\tilde{f}_{k} \longrightarrow f\left(\cdot, \tilde{y}_{1}+\bar{z}_{1}\right) \quad \text { a.s. in } \Omega
$$

with values in $L^{2}\left(0, T ; L^{2}(G)\right)$. The same applies for $\tilde{g}_{k}$.

Set

$$
\begin{gathered}
u=-\int_{0}^{t} f\left(s, \bar{z}_{1}\right) d s-\int_{0}^{t} g\left(s, \bar{z}_{1}\right) d s, \\
\left\{\bar{y}_{1}, \bar{y}_{2}\right\}=\left\{\tilde{y}_{1}+\bar{z}_{1}, \tilde{y}_{2}+\bar{z}_{2}\right\},
\end{gathered}
$$




\section{Exact controllability}

then $\{u, \bar{v}, \overline{\mathbf{y}}\}$ is a solution of (1.1)-(1.4). The estimates of the theorem are now immediate consequences of those of Lemmas 3.5, 3.8.

\section{References}

[1] A. Bensoussan and R. Temam, Equations stochastiques du type Navier-Stokes, Journal of Functional Analysis 13 (1973), no. 2, 195-222.

[2] F. E. Browder, Nonlinear accretive operators in Banach spaces, Bulletin of the American Mathematical Society 73 (1967), 470-476.

[3] T. Kato, Accretive operators and nonlinear evolution equations in Banach spaces, Nonlinear Functional Analysis (Proceedings of Symposia in Pure Mathematics, vol. 18, Part 1, Chicago, Ill, 1968), American Mathematical Society, Rhode Island, 1970, pp. 138-161.

[4] J.-L. Lions, Exact controllability, stabilization and perturbations for distributed systems, SIAM Review 30 (1988), no. 1, 1-68.

[5] D. L. Russell, Controllability and stabilizability theory for linear partial differential equations: recent progress and open questions, SIAM Review 20 (1978), no. 4, 639-739.

[6] B. A. Ton, Exact controllability for a semilinear wave equation with both interior and boundary controls, Abstract and Applied Analysis 2005 (2005), no. 6, 619-637.

[7] ___ Exact controllability of a nonlinear stochastic wave equation with measure data, submitted for publication.

[8] E. Zuazua, Exact boundary controllability for the semilinear wave equation, Nonlinear Partial Differential Equations and Their Applications. Collège de France Seminar, vol. 10 (Paris, 19871988), Pitman Research Notes in Mathematics Series, vol. 220, Longman Scientific \& Technical, Harlow, 1991, pp. 357-391.

Bui An Ton: Department of Mathematics, The University of British Columbia,

Vancouver, BC, Canada V6T 1 Z2

E-mail address: bui@math.ubc.ca 


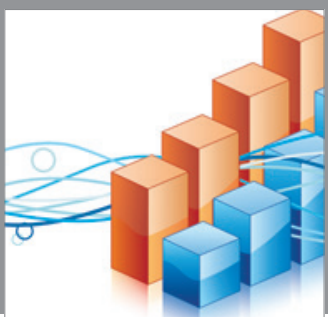

Advances in

Operations Research

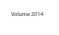

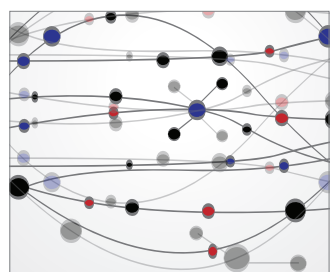

\section{The Scientific} World Journal
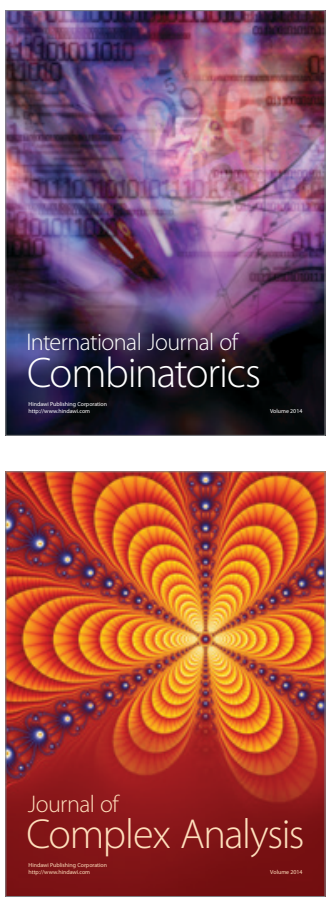

International Journal of

Mathematics and

Mathematical

Sciences
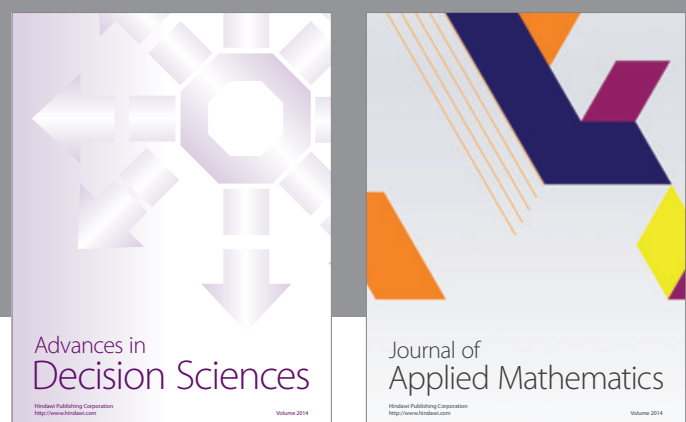

Journal of

Applied Mathematics
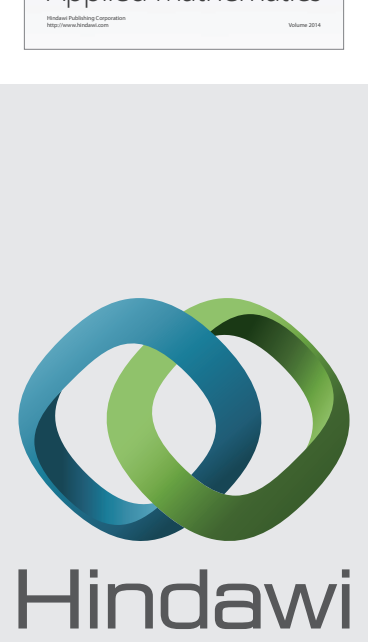

Submit your manuscripts at http://www.hindawi.com
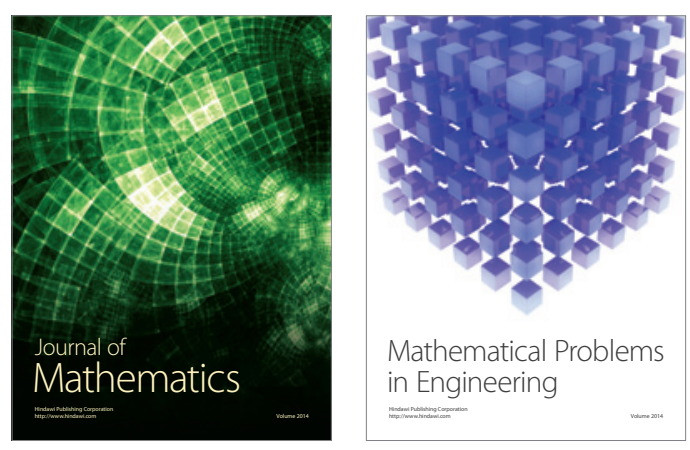

Mathematical Problems in Engineering
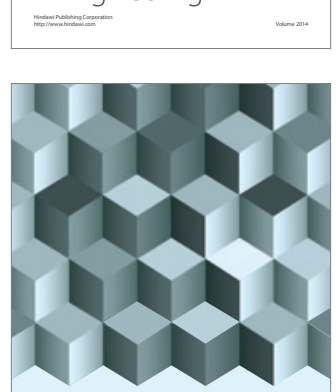

Journal of

Function Spaces
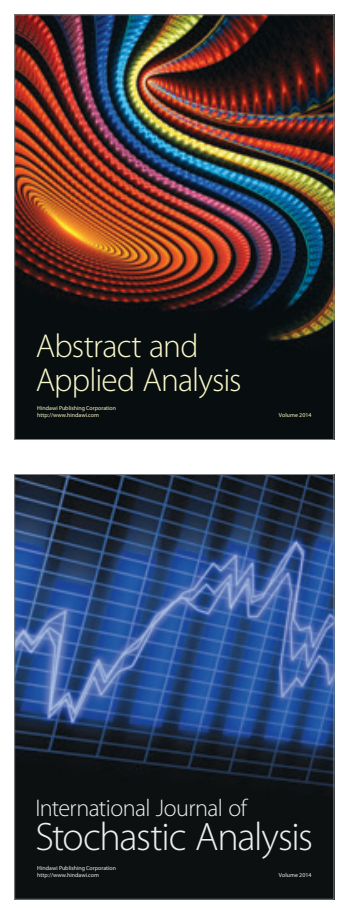

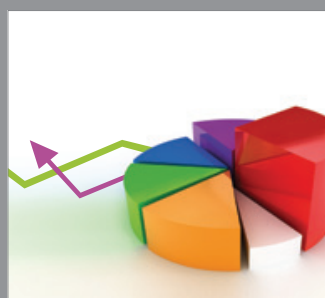

ournal of

Probability and Statistics

Promensencen
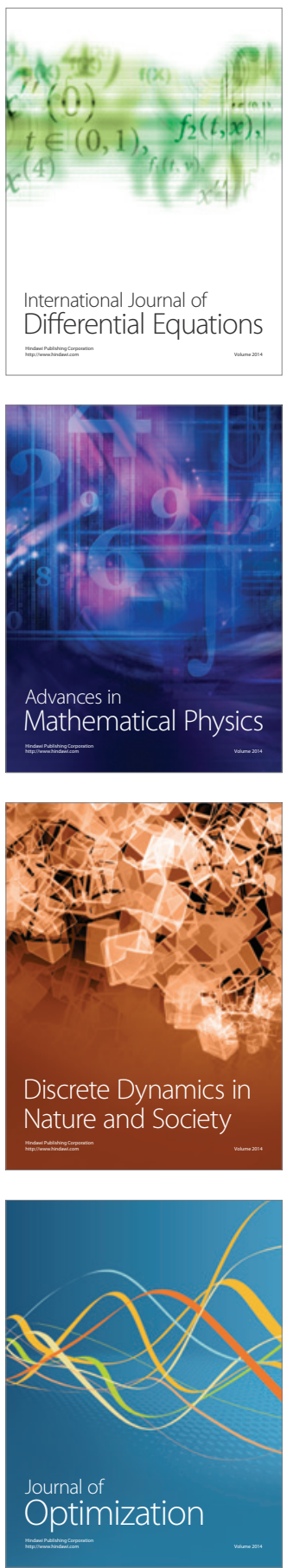